\title{
Characterization and antimicrobial activity of Streptomyces strains from soils in southern Thailand
}

\author{
Paranee Sripreechasak ${ }^{1}$, Khanit Suwanborirux ${ }^{2}$ and Somboon Tanasupawat ${ }^{1}$ \\ ${ }^{1}$ Department of Biochemistry and Microbiology, Faculty of Pharmaceutical Sciences, Chulalongkorn University, Bangkok 10330, Thailand. \\ ${ }^{2}$ Department of Pharmacognosy and Pharmaceutical Botany, Faculty of Pharmaceutical Sciences, Chulalongkorn University, Bangkok 10330, Thailand.
}

\begin{tabular}{l} 
ARTICLE INFO \\
\hline Article history: \\
Received on: 02/08/2014 \\
Revised on: 16/08/2014 \\
Accepted on: 09/09/2014 \\
Available online: $30 / 10 / 2014$ \\
\hline Key words: \\
Actinomycetes, Streptomyces, \\
antimicrobial activity, soil
\end{tabular}

\begin{abstract}
Streptomyces strains are superior to other actinomycete strains in their ability to produce large numbers and varieties of antibiotics. The aim of this research was to study on the identification and antimicrobial activity of Streptomyces strains which were isolated from thirteen soil samples collected around Angthong Islands National Park, Surat Thani province, the southern part of Thailand. Twenty-six strains were isolated by using the dilution plating method on starch casein nitrate agar plate and potato starch-glycerol agar plate. On the basis of phenotypic characteristics and the $16 \mathrm{~S}$ rRNA gene sequence analysis, they were belonged to the genus Streptomyces and were identified as $S$. tendae (5 isolates), S. malachitospinus (2 isolates), S. marokkonensis (2 isolates), S. parvulus (2 isolates), S. fragilis (1 isolate), S. diastaticus (1 isolate), S. drozdowiczii (1 isolate), $S$. olivochromogenes (1 isolate), S. aureus (2 isolates), S. iranensis (1 isolate), S. rapamycinicus (1 isolate), $S$. yatensis (1 isolate), S. samsunensis (4 isolates) and $S$. spiralis (2 isolates). On the antimicrobial activity screening, 15 isolates exhibited activity against Bacillus subtilis ATCC 6633, 13 isolates against Kocuria rhizophila ATCC 9341, 6 isolates against Mucor racemosus IFO 4581 and Candida albicans KF1, one isolate against Escherichia coli NIHJ KB213 and 5 isolates against Xanthomonas campestris pv. oryzae KB88.
\end{abstract}

\section{INTRODUCTION}

Streptomycetes are environmental filamentous Grampositive bacteria of great commercial value. They have been well known as the producers of various useful bioactive metabolites particularly the antibiotics (Goodfellow et al., 1988). The genus Streptomyces belonging to the family Steptomycetaceae was an aerobic, Gram-positive, spore-forming actinomycetes (Kämpfer, 2012). Strains of the genus Streptomyces have been well known as the producers of various useful bioactive metabolites particularly the antibiotics including erythromycin, tetracycline, streptomycin, chloramphenicol, neomycin, nystatin, amphotericin, kanamycin and cycloheximide (Glasby, 1993; Berdy, 2005). Streptomyces strain represents a group of microorganisms widely distributed in nature. Streptomyces strains

\footnotetext{
* Corresponding Author

Somboon Tanasupawat, Department of Biochemistry and Microbiology, Faculty of Pharmaceutical Sciences, Chulalongkorn University, 254 Phayathai Road, Wangmai, Bangkok 10330, Thailand.

E-mail:Somboon.T@chula.ac.th,tanalab@yahoo.com
}

remains a focus of systematic research because they are still a rich source of commercially significant compounds such as antibiotics, enzymes, enzyme inhibitors and pharmacologically active agents (Goodfellow et al., 1988). In this study, we focused to isolate and identify of Streptomyces strains and screen of antimicrobial activity of Streptomyces isolates from soils in the southern part of Thailand.

\section{MATERIALS AND METHODS}

\section{Characterization of the isolates}

\section{Isolation and phenotypic characterization of the isolates}

Actinomycete strains were isolated from thirteen soil samples collected around Angthong Islands National Park, Surat Thani province, the southern part of Thailand. The dried soil samples were suspended in distilled water and heated at $55^{\circ} \mathrm{C}$ for 5 minutes. Serial dilutions of the suspension were prepared by the 10 fold dilution method. $10^{-2}$ and $10^{-3}$ dilutions of the suspension, aliquots of $0.1 \mathrm{ml}$ were spreaded on surface of potato starchglycerol agar (potato starch $1.0 \%$, glycerol $1.0 \%, \mathrm{~K}_{2} \mathrm{HPO}_{4} 0.2 \%$, $\left(\mathrm{NH}_{4}\right)_{2} \mathrm{SO}_{4} 0.2 \%, \mathrm{MgSO}_{4} .7 \mathrm{H}_{2} \mathrm{O} 0.1 \%, \mathrm{NaCl}_{2} 0.1 \%, \mathrm{CaCO}_{3} 0.2 \%$ 
and agar 1.2\%, pH 7.0) (Tajima et al., 2001) and starch casein nitrate agar (starch $1.0 \%$, sodium caseinate $0.03 \%, \mathrm{KNO}_{3} 0.2 \%$ and agar $1.5 \%, \mathrm{pH} 7.0$ ) (Seong, 2001) supplemented with $25 \mathrm{mg} \mathrm{l}^{-1}$ of nystatin for antifungal. Each agar plate was supplemented with $10 \mathrm{mg} \mathrm{l}^{-1}$ of tetracycline and/or $50 \mathrm{mg} \mathrm{l}^{-1}$ of novobiocin.

The different colonies were picked up and streaked for further purification on International Streptomyces Project (ISP) media, ISP 2 (Shirling and Gottlieb, 1966). The pure isolates were observed for their cultural characteristics after cultivation on ISP 2 and ISP 3 agar at $28{ }^{\circ} \mathrm{C}$ for 2 weeks. The colors of substrate, aerial mycelium and soluble pigments were determined using the NBS/IBCC color chart (Mundie, 1995). Morphological observation was done by using a light microscope and scanning electron microscope (JSM-5410LV, Japan) on the culture grown on ISP 2, ISP 3, ISP 4 or YS (Yeast extract-starch) agar plates at $30{ }^{\circ} \mathrm{C}$ for 14 days. The phenotypic characteristics of isolates were determined as described by Shirling and Gottlieb (1966) and Arai (1975).

\section{S rRNA gene sequencing and phylogenetic analysis}

Genomic DNA of each isolates was prepared by sonication of the cell suspension (Matsumoto et al., 2006) cultured in YD broth (1.0\% yeast extract and $1.0 \%$ dextrose). The $16 \mathrm{~S}$ rRNA gene was amplified using the primers described by Takahashi et al. (2002). The reaction was carried out in a $50 \mu \mathrm{l}$ reaction volume consisting of $5.0 \mu \mathrm{l}$ of DNA solution, 29.6 of sterile Milli Q water, $5.0 \mathrm{ml}$ of 10x Taq buffer, $2 \mathrm{ml}$ of dNTP, 4.0 $\mathrm{ml}$ of each primer in primer pairs (11F \& 925R), $0.4 \mu \mathrm{L}$ of Ex Taq DNA polymerase. DNA thermal cycler was use for $16 \mathrm{~S}$ rRNA gene amplification using the temperature profile of initial denaturation at $95{ }^{\circ} \mathrm{C}$ for $1 \mathrm{~min}$, followed by 30 cycles of denaturation at $95{ }^{\circ} \mathrm{C}$ for $1 \mathrm{~min}$, primer annealing at $50{ }^{\circ} \mathrm{C}$ for 1 min, extension at $72{ }^{\circ} \mathrm{C}$ for $1.5 \mathrm{~min}$, and a final extension at $72{ }^{\circ} \mathrm{C}$ for $2 \mathrm{~min}$. The PCR products were checked by agarose gel electrophoresis. The PCR products were sequenced on a DNA sequencer (Applied Biosystems 3130 Genetic Analyzer) using a BigDye Terminator v3.1 cycle Sequencing kit (Applied Biosystems), according to the manufacturer's instructions.

The obtained sequence was compared with all sequences from GenBank using the BLAST program. The clustalw2 program was used for multiple alignments with selected sequences for calculating evolution distances (Kimura, 1980) by Sea View version 4.2 (Gouy et al., 2010). The phylogenetic tree was constructed using the neighbor-joining method (Saitou and Nei, 1987). Data were resampled with 100 bootstrap replications. The values for sequence similarities among the most closely related strains were determined using the EzTaxon-e server (Kim et al., 2012).

\section{Antimicrobial activities of the isolates}

Each isolate was cultured in $10 \mathrm{ml}$ of 301 seed medium (2.4\% starch, $0.1 \%$ glucose, $0.3 \%$ peptone, $0.3 \%$ meat extract, $0.5 \%$ yeast extract, $0.4 \% \mathrm{CaCO}_{3}, \mathrm{pH} 7.0$ ) and cultivated on shaker (200 rpm) at $28{ }^{\circ} \mathrm{C}$ for 3-7 days. One percentage of seed culture was transferred into $10 \mathrm{ml}$ of 51 medium $(0.5 \%$ glucose, $0.5 \%$ corn steep powder, $1.0 \%$ oatmeal, $1.0 \%$ pharmamedia, $0.5 \%$ $\mathrm{K}_{2} \mathrm{HPO}_{4}, 0.5 \% \mathrm{MgSO}_{4} \cdot 7 \mathrm{H}_{2} \mathrm{O}, 1 \mathrm{ml} / \mathrm{l}$ trace metal solution, tap water) and 53 medium (2.0\% soluble starch, $2.0 \%$ glycerol, 2.0 $\%$ nutrient broth, $1.0 \%$ defatted wheat germ, $0.3 \% \mathrm{CaCO}_{3}$, tap water) and cultivated on shaker $(200 \mathrm{rpm})$ at $28{ }^{\circ} \mathrm{C}$ for 6 days. The 6-day-cultured broth was extracted with $10 \mathrm{ml}$ of ethanol by shaking (200 rpm) for $30 \mathrm{~min}$, mixed well and centrifuged $(3,000$ rpm) for $5 \mathrm{~min}$.

Supernatant $(50 \%$ ethanol extract) was examined antimicrobial assay against microorganisms, Kocuria rhizophila ATCC 9341, Bacillus subtilis ATCC 6633, Escherichia coli NIHJ KB213, Xanthomonas campestris pv. oryzae KB88, Candida albicans KF1 and Mucor racemosus IFO 4581 by a paper disc diffusion assay (Lorain, 1991).

\section{RESULTS AND DISCUSSION}

\section{Characterization of the isolates}

Twenty-six strains were isolated from thirteen soil samples collected around Angthong Islands National Park, Surat Thani province, the southern part of Thailand. On the basis of morphological, cultural, physiological and biochemical characteristics including the $16 \mathrm{~S}$ rRNA gene sequence analysis, they were belonged to the genus Streptomyces (Kämpfer, 2012). The phylogenetic tree (Figure 1) based on 16S rRNA gene sequences similarity showed that they were located within the lineage of the genus Streptomyces. The isolates showed good growth and formed extensively branched substrate mycelia and spores on ISP 2 and ISP 3 agar plates. Their morphological on ISP 2, ISP 3, ISP 4 or YS (Yeast extract-starch) agar plates are shown in Figure 2.

The cultural characteristics such as upper colony, aerial mass color, reversed colonial appearance and soluble pigment are described in Tables 1 and 2. All isolates grew at pH 5-11 and they hydrolysed starch, but did not hydrolyse gelatin. Most of them reduced nitrate to nitrite and some showed peptonization/ coagulation. Some grew on $4-10 \% \mathrm{NaCl}$. The utilization of carbon sources was variable (Table 3 ). The details of their phenotypic characteristics are listed in Tables 1, 2 and 3.

Group 1 contained 5 isolates (Table 4). The calculated pair-wise sequence similarities of isolates $\mathrm{KC}-054, \mathrm{KC}-066, \mathrm{KC}$ 074, KC-075 and KC-076 were $100 \%$ to S. tendae ATCC $19812^{\mathrm{T}}$ (Figure 1). They were identified as $S$. tendae (Syed et al., 2007).

Group 2 contained 2 isolates, KC-060 and KC-088 (Table 4). The calculated pair-wise sequence similarities of isolates were 99.8 and $99.9 \%$, respectively to S. malachitospinus NBRC $101004^{\mathrm{T}}$. They were identified as $S$. malachitospinus (Kalakoutskii et al., 1990).

Group 3 contained 2 isolates, KC-073 and KC-155 (Table 4). The calculated pair-wise sequence similarity of them was $99.4 \%$ to $S$. marokkonensis $\mathrm{Ap}^{\mathrm{T}}{ }^{\mathrm{T}}$. These strains were identified as S. marokkonensis (Bouizgarne et al., 2009). 
Group 4 contained 2 isolates, KC-055 and $\mathrm{KC}-090$ (Table 4). The calculated pair-wise sequence similarities of $\mathrm{KC}$ 055 and KC-090 were $100 \%$ to $S$. parvulus NBRC $13193^{\mathrm{T}}$. They were identified as $S$. parvulus NBRC $13193^{\mathrm{T}}$ (Reddy et al., 2011).

Group 5 contained 1 isolate, $\mathrm{KC}-072$ (Table 4). The calculated pair-wise sequence similarity of $\mathrm{KC}-072$ was $99.3 \%$ to S. fragilis NRRL $2424^{\mathrm{T}}$. This strain was identified as $S$. fragilis (Santhanam et al., 2013).

Group 6 contained 1 isolate, KC-070 (Table 4). The calculated pair-wise sequence similarity of $\mathrm{KC}-070$ was $99.7 \%$ to S. diastaticus subsp. ardesiacus NRRL B-1773 ${ }^{\mathrm{T}}$. This strain was identified as $S$. diastaticus subsp. ardesiacus (Arumugam et al., 2011).

Group 7 contained 1 isolate, KC-085 (Table 4). The calculated pair-wise sequence similarity of $\mathrm{KC}-085$ was $100 \%$ to S. drozdowiczii NBRC $101007^{\mathrm{T}}$ (Figure 1). This strain was identified as $S$. drozdowiczii (Semedo et al., 2004).

Group 8 contained 1 isolate, KC-156 (Table 4). The calculated pair-wise sequence similarity of the isolate was $98.4 \%$ to $S$. olivochromogenes NBRC $3178^{\mathrm{T}}$ (98.4). It was identified as $S$. olivochromogenes (Sripreechasak et al., 2013a).

Group 9 contained 2 isolates, KC-141 and KC-142 (Table 4). The calculated pair-wise sequence similarities of them was $99.5 \%$ and $99.6 \%$ to $S$. aureus NBRC $100912^{\mathrm{T}}$. These strains were identified as $S$. aureus (Manfio et al., 2003).

Group 10 contained 1 isolate, KC-087 (Table 4). The calculated pair-wise sequence similarity of $\mathrm{KC}-087$ was $99.8 \%$ to S. iranensis $\mathrm{HM} 35^{\mathrm{T}}$. This strain was identified as $S$. iranensis (Hamedi et al., 2010).

Group 11 contained 1 isolate, $\mathrm{KC}-058$ (Table 4). The calculated pair-wise sequence similarity of KC-058 was $99.3 \%$ to S. rapamycinicus NRRL B-5491 ${ }^{\mathrm{T}}$. This strain was identified as $S$. rapamycinicus (Kumar and Goodfellow, 2008).

Group 12 contained 1 isolate, KC-079 (Table 4). The calculated pair-wise sequence similarity of KC-079 was $99.4 \%$ to S. yatensis $\mathrm{NBRC} 101000^{\mathrm{T}}$. This strain was identified as $S$. yatensis (Saintpierre et al., 2003).

Group 13 contained 4 isolates, KC-080, KC-093, KC-094 and $\mathrm{KC}-151$ (Table 4). The calculated pair-wise sequence similarities of KC-080, KC-093, KC-094 and KC-151 were 99.9$100 \%$ to $S$. samsunensis M1463 ${ }^{\mathrm{T}}$. These strains were identified as S. samsunensis (Sazak et al., 2011).

Group 14 contained 2 isolates, $\mathrm{KC}-062$ and $\mathrm{KC}-063$ (Table 4). The calculated pair-wise sequence similarities of $\mathrm{KC}$ 062 and KC-063 were 99.0-99.4\% to S. spiralis NBRC $14215^{\mathrm{T}}$. These strains were identified as S. spiralis (Kämpfer, 2012).

\section{Antimicrobial activities of the isolates}

On the antimicrobial activitiy screening, based on the cultivation in 51 medium and 53 medium (Table 4), Group 1 isolates, KC-066 and KC-075 identified as S. tendae and Group 3 isolates, $\mathrm{KC}-073$ and $\mathrm{KC}-155$ identified as $S$. marokkonensis showed antibacterial activity against B. subtilis and $K$. rhizophila strain when fermented in both media. Group 2 isolate, KC-060 identified as S. malachitospinus and Group 14 isolates, KC-062 and KC-063 identified as S. spiralis could inhibit B. subtilis, $K$. rhizophila or $X$. campestris strain when fermented in 53 medium. Group 4 isolates, KC-055 and KC-090 exhibited against $B$. subtilis, $K$. rhizophila and $X$. campestris strain when fermented in both media.

The isolates in Group 5, 6 and 7 showed no antimicrobial activity on the tested strains. Group 8 isolate, KC-156 identified as $S$. olivochromogenes showed antibacterial activity against $B$. subtilis and $K$. rhizophila strain when fermented in both media and it inhibited $E$. coli when fermented in 53 medium. Group 9 isolate, KC-141 identified as $S$. aureus showed antibacterial activity against B. subtilis and K. rhizophila strain when fermented in 51 medium.

Group 10 isolate, KC-087 identified as S. iranensis showed antibacterial activity against $B$. subtilis, $K$. rhizophila, $C$. albicans and $M$. racemosus strain when fermented in 53 medium. Group 11, Group 12 and Group 13 isolates showed antibacterial activity against $B$. subtilis, $K$. rhizophila, $C$. albicans and $M$. racemosus or $X$. campestris strain when fermented in both media. This study, S. tendae, S. malachitospinus, S. marokkonensis, S. parvulus, $S$. fragilis, $S$. diastaticus, $S$. drozdowiczii, $S$. olivochromogenes, S. aureus, S. iranensis, S. rapamycinicus, S. yatensis, S. samsunensis, and S. spiralis were distributed in soil samples collected around Angthong Islands National Park, Surat Thani province, the southern Thailand.

The $S$. tendae strains have been reported to produce nikkomycin (Roos et al., 1992); cyclohexenylglycine, an isoleucine antagonist with antibacterial activity; the naphthoquinone compound juglomycin, which had antitumor activity and chlorothricin; a glycosylated macrolide antibiotic that acted as an antagonist of acetyl-coenzyme A in bacteria and antifungal, chitin-binding protein (Bormann et al., 1999). S. parvulus strain produced polypeptide antibiotic and showed antibacterial activity (Shetty et al., 2014).

The strain of S. fragilis produced azaserine and 6-diazo5-oxo-L-norleucine (DON) (Pittillo and Hunt, 1967) while the strains of $S$. diastaticus have been reported to produce two polyene macrolide antibiotics, rimocidin and CE-108 (Seco et al., 2005) and antifungus, oligomycins A and C (Yang et al., 2010). S. olivochromogenes produced 4-hydroxy-3-methoxycinnamic (ferulic) acid esterase (Faulds and Williamson, 1991) whereas the strain S. rapamycinicus strain NRRL 5491 was reported to produce the important drug rapamycin (Baranasic et al., 2013).

In southern Thailand, actinobacteria isolated from soils collected in Krung Ching Waterfall National Park, Nakhon Si Thammarat were reported to be Streptomyces exfoliates, $S$. vinaceusdrappus; $S$. tendae, $S$. aureus, $S$. atriruber, $S$. olivochromogenes, $\quad S$. malaysiensis, $S$. purpeofuscus, $S$. sparsogenes, $S$. aldersoniae, $S$. rapamycinicus, and $S$. youssoufiensis; Nocardia niigatensis; Amycolatopsis rifamycinica; Kitasatospora saccharophila; Rhodococcus triatomae; and Gordonia alkanivorans (Sripreechasak et al., 2013b). 


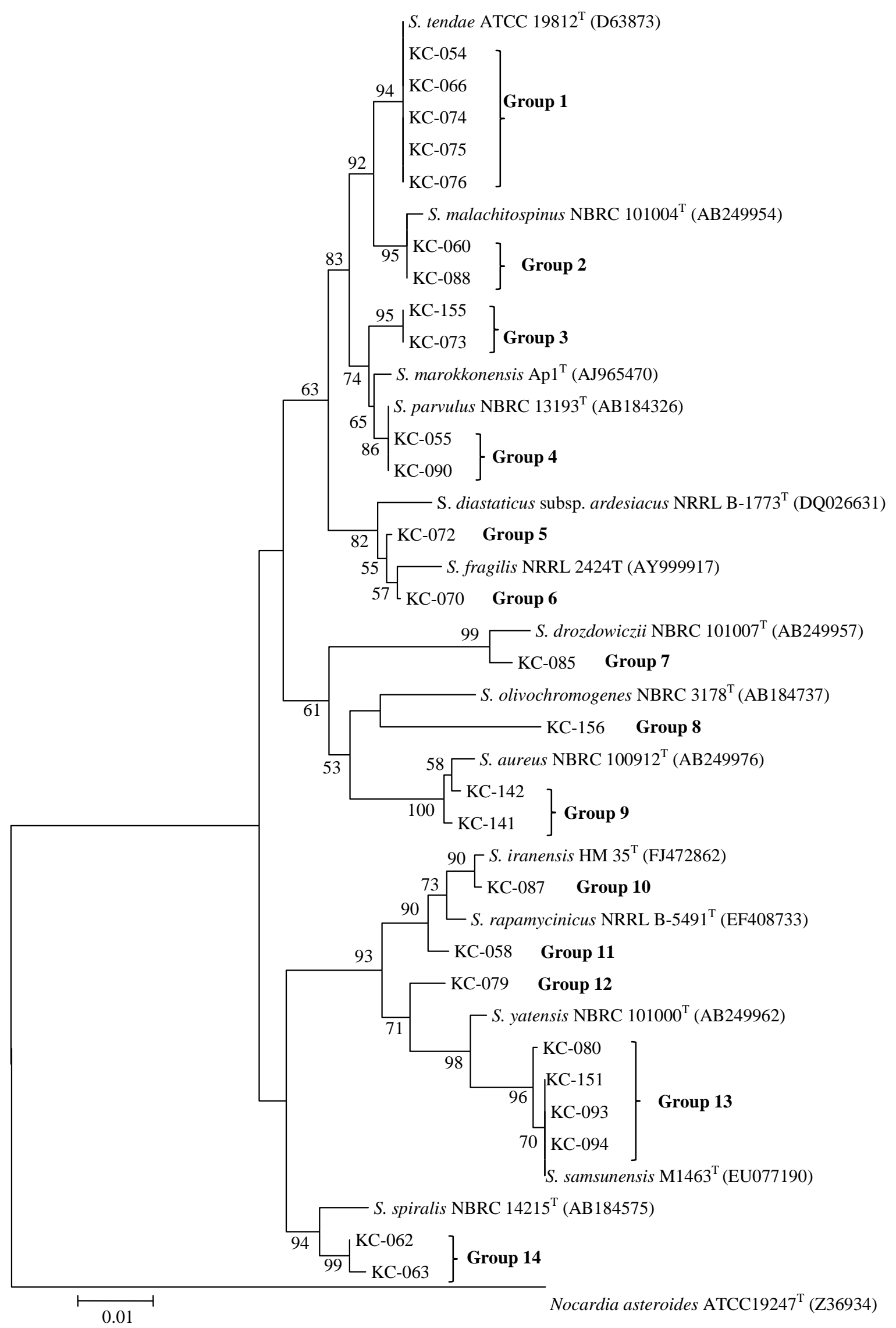

Fig. 1: Neighbor-joining tree based on 16S rRNA gene sequences showing relationship between 26 isolates and closely related type strains of the genus Streptomyces. Only bootstrap values above $50 \%$ (percentages of 1000 replications) are indicated. Bar, 0.01 nucleotide substitutions per site. 


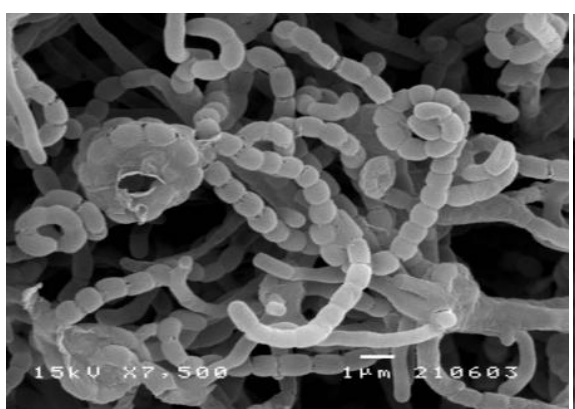

1. KC-075 on ISP 3

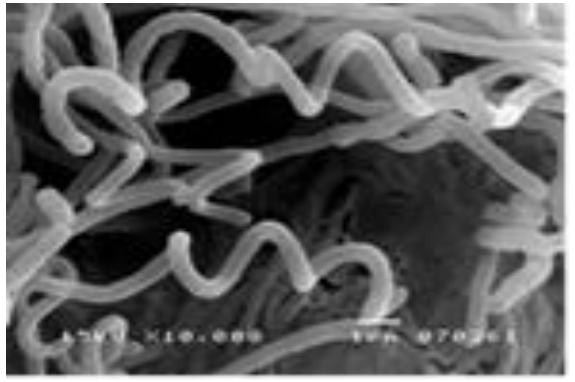

4. KC-055 on ISP 4

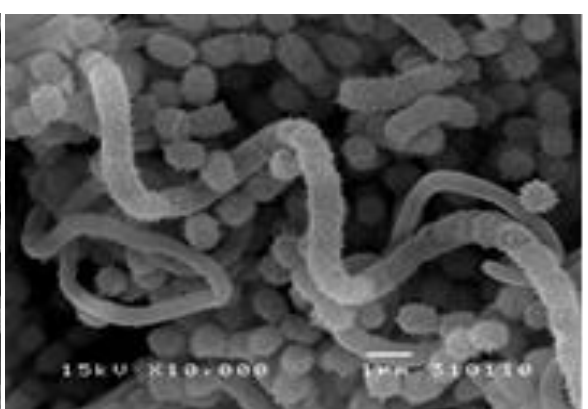

2. KC-088 on ISP 4

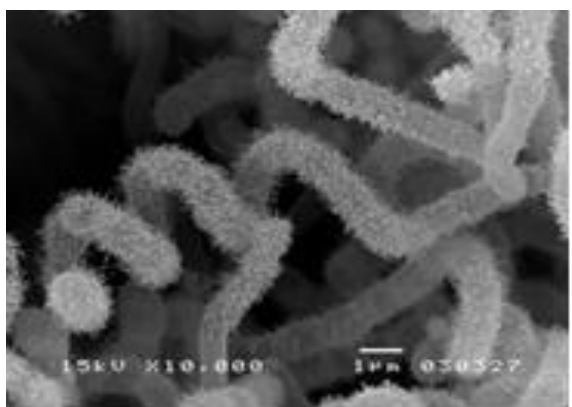

3. KC-155 on ISP 4

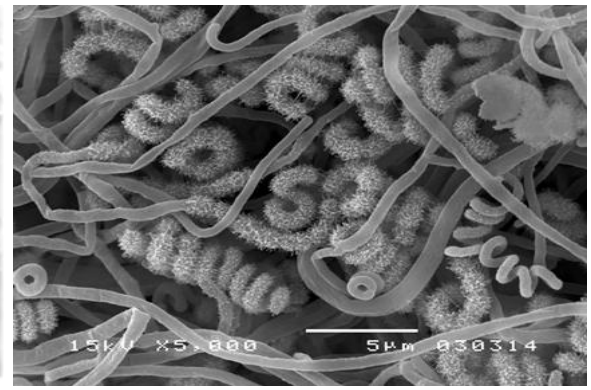

5. KC-072 on ISP 2

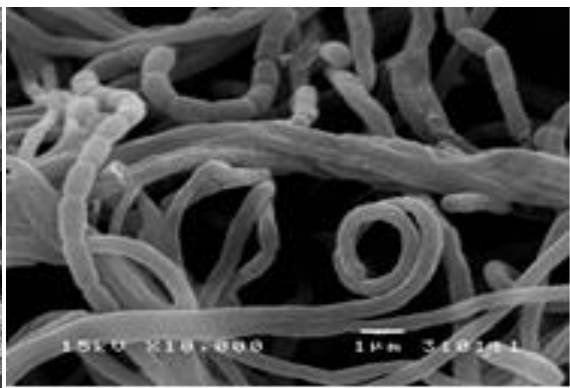

6. $\mathrm{KC}-070$ on ISP 4

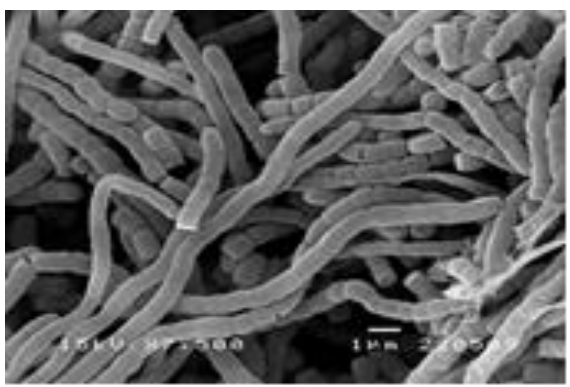

7. KC-085 on ISP 4

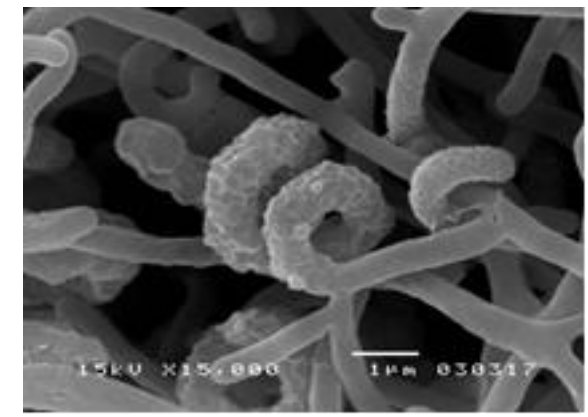

10. KC-087 on ISP 4

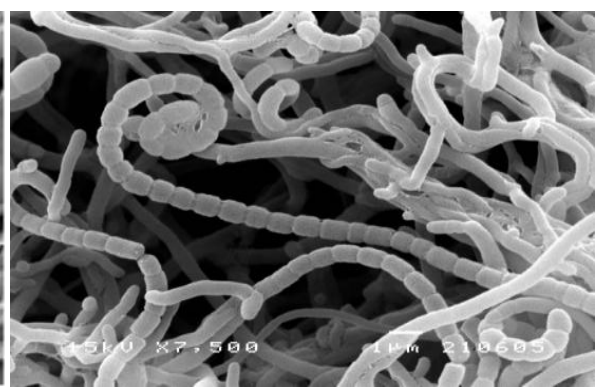

8. KC-156 on ISP 3

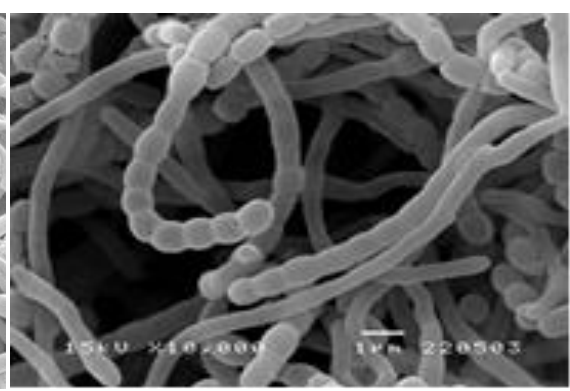

9. KC-141 on ISP 4

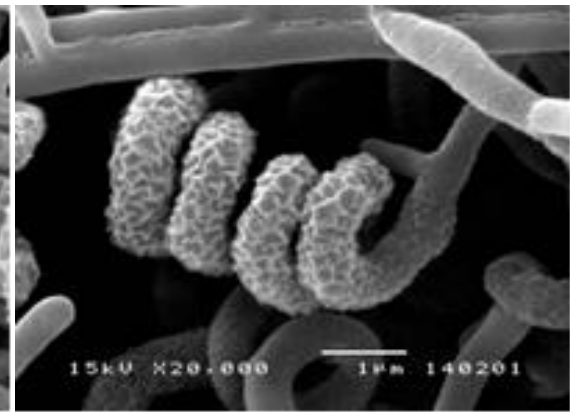

12. $\mathrm{KC}-079$ on ISP 4

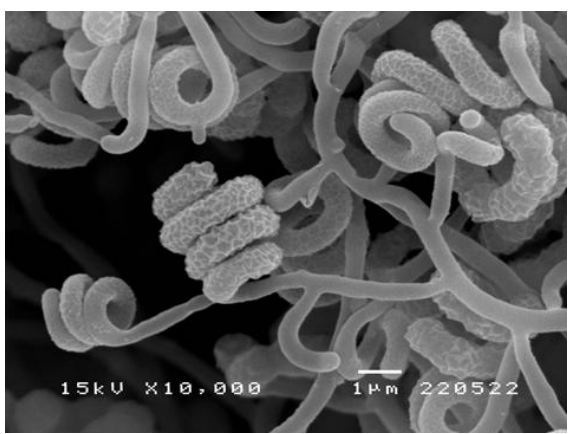

13. KC-080 on ISP 4

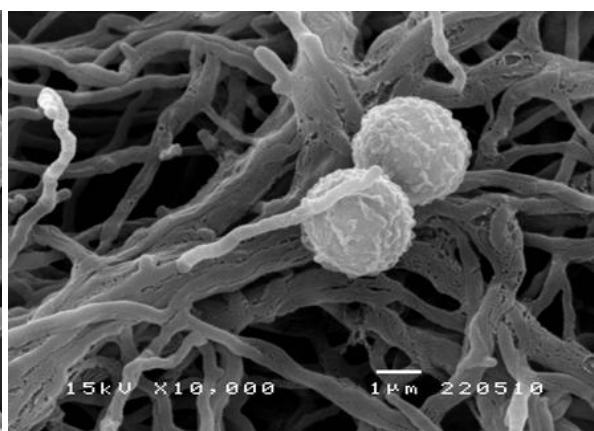

14. KC-062 on YS

Fig. 2: Scanning electron micrograph of isolates in Group 1 to Group 14, grown on ISP 2, ISP3, ISP4 or YS agar plates at $30{ }^{\circ} \mathrm{C}$ for 2 weeks. 
Table 1: Cultural characteristics of Streptomyces isolates in Group 1 to Group 14 on ISP 2 agar .

\begin{tabular}{|c|c|c|c|c|c|}
\hline Group & Growth & Upper Colony & Aerial mass color & Reverse colony & Soluble pigment \\
\hline 1 & Good & $\begin{array}{l}\text { Pale greenish yellow - light } \\
\text { greenish yellow }\end{array}$ & Abundant, white - bluish gray & $\begin{array}{l}\text { Pale greenish yellow - } \\
\text { moderate olive }\end{array}$ & None \\
\hline 2 & Good & $\begin{array}{l}\text { Pale yellow - grayish greenish } \\
\text { yellow }\end{array}$ & None & Pale yellow - grayish greenish yellow & None \\
\hline 3 & Good & Pale yellow & None & Pale yellow - pale yellowish green & None \\
\hline 4 & Good & $\begin{array}{l}\text { Pale yellow - grayish greenish } \\
\text { yellow }\end{array}$ & None & $\begin{array}{l}\text { Pale yellow - grayish greenish } \\
\text { yellow }\end{array}$ & None \\
\hline 5 & Good & Grayish greenish yellow & $\begin{array}{l}\text { Abundant, white - brownish } \\
\text { black }\end{array}$ & Light grayish olive - grayish olive & None \\
\hline 6 & Good & $\begin{array}{l}\text { Brownish pink - light reddish } \\
\text { brown }\end{array}$ & Abundant, white - medium gray & $\begin{array}{l}\text { Dark grayish yellow - grayish reddish } \\
\text { brown }\end{array}$ & None \\
\hline 7 & Good & Colorless & Abundant, bluish gray & Light grayish olive - brownish black & $\begin{array}{l}\text { Dark grayish } \\
\text { yellow }\end{array}$ \\
\hline 8 & Good & Strong brown & Abundant, light gray & Light brown - dark brown & Light brown \\
\hline 9 & Good & Pale yellow & None & Pale yellow & None \\
\hline 10 & Good & Pale yellow & Abundant, white - bluish gray & Dark yellow & Grayish yellow \\
\hline 11 & Good & Dark grayish yellow & $\begin{array}{l}\text { Abundant, greenish white - } \\
\text { greenish black }\end{array}$ & Light grayish olive - grayish olive & None \\
\hline 12 & Good & Colorless - pale yellow & $\begin{array}{l}\text { Abundant, greenish white - } \\
\text { grayish olive }\end{array}$ & Light yellow - dark olive brown & None \\
\hline 13 & Good & Colorless - light olive gray & $\begin{array}{l}\text { Abundant, greenish white - } \\
\text { medium gray }\end{array}$ & Light yellow - light olive gray & None - deep yellow \\
\hline 14 & Good & Strong greenish yellow & Abundant, white & Strong greenish yellow & None \\
\hline
\end{tabular}

Table 2: Cultural characteristics of Streptomyces isolates in Group 1 to Group 14 on ISP 3 agar.

\begin{tabular}{|c|c|c|c|c|c|}
\hline Group & Growth & Upper colony & Aerial mass color & Reverse colony & Soluble pigment \\
\hline 1 & Good & $\begin{array}{l}\text { Pale greenish yellow - } \\
\text { grayish greenish yellow }\end{array}$ & Abundant, white - bluish gray & $\begin{array}{l}\text { Pale greenish yellow - } \\
\text { moderate yellow }\end{array}$ & None \\
\hline 2 & Good & $\begin{array}{l}\text { Pale yellowish green - } \\
\text { grayish yellow }\end{array}$ & $\begin{array}{l}\text { Abundant, white - light greenish } \\
\text { gray }\end{array}$ & $\begin{array}{l}\text { Pale yellowish green - light grayish } \\
\text { yellowish brown }\end{array}$ & $\begin{array}{l}\text { None - pale orange } \\
\text { yellow }\end{array}$ \\
\hline 3 & Good & $\begin{array}{l}\text { Yellowish white -pale } \\
\text { orange yellow }\end{array}$ & Moderate, greenish white & Yellowish white - pale orange yellow & None \\
\hline 4 & Good & $\begin{array}{l}\text { Pale yellowish green - } \\
\text { strong greenish yellow }\end{array}$ & $\begin{array}{l}\text { Abundant, greenish white - light } \\
\text { greenish gray }\end{array}$ & Pale yellowish green - moderate olive & $\begin{array}{l}\text { Pale yellowish green } \\
\text { - light greenish } \\
\text { yellow }\end{array}$ \\
\hline 5 & Good & $\begin{array}{l}\text { Grayish yellow - dark } \\
\text { grayish yellow }\end{array}$ & $\begin{array}{l}\text { Abundant, light greenish gray - } \\
\text { greenish gray }\end{array}$ & $\begin{array}{l}\text { Dark grayish yellow - } \\
\text { dark olive brown }\end{array}$ & Grayish yellow \\
\hline 6 & Good & Moderate reddish brown & Abundant, white - purplish gray & Very dark red - light grayish red & None \\
\hline 7 & Good & Moderate olive brown & Abundant, bluish gray & Dark olive brown & Grayish yellow \\
\hline 8 & Good & Grayish greenish yellow & Abundant, light gray & Dark grayish yellow & None \\
\hline 9 & Good & Moderate brown & Abundant, light gray & Deep brown - dark brown & Strong brown \\
\hline 10 & Good & Pale yellow & Abundant, white - medium gray & Moderate yellow & Grayish yellow \\
\hline 11 & Good & Grayish greenish yellow & $\begin{array}{l}\text { Abundant, greenish white - } \\
\text { greenish black }\end{array}$ & $\begin{array}{l}\text { Grayish olive green - } \\
\text { dark grayish olive green }\end{array}$ & None \\
\hline 12 & Good & $\begin{array}{l}\text { Pale yellowish green - } \\
\text { light grayish olive }\end{array}$ & Abundant, white - greenish black & Light grayish olive - olive gray & None \\
\hline 13 & Good & Colorless - olive gray & $\begin{array}{l}\text { Abundant, greenish white - light } \\
\text { greenish gray }\end{array}$ & Light yellowish green - olive gray & None - dark yellow \\
\hline 14 & Good & $\begin{array}{l}\text { Moderate greenish yellow - } \\
\text { deep yellowish pink }\end{array}$ & Abundant, white & Pale greenish yellow - deep pink & None \\
\hline
\end{tabular}

Table 3: Differential characteristics of Streptomyces isolates in Group 1 to Group 14.

\begin{tabular}{|c|c|c|c|c|c|c|c|c|c|c|c|c|c|c|}
\hline Characteristics & 1 & 2 & 3 & 4 & 5 & 6 & 7 & 8 & 9 & 10 & 11 & 12 & 13 & 14 \\
\hline Nitrate reduction & + & + & $-(+1)$ & - & - & + & + & + & $+(-1)$ & + & - & - & + & - \\
\hline Peptonization/Coagulation & $-1-$ & $-1-$ & $+(-1)$ & $-1+$ & $-1-$ & $-1-$ & $+/-$ & $-1-$ & $-(+)$ & w/- & $-1-$ & $+/-$ & $+1-$ & - \\
\hline $\mathrm{NaCl}$ tolerance $(\%)$ & $7-9$ & 8 & $4-5$ & 8 & 10 & 10 & 8 & 8 & 7 & 7 & 4 & 4 & 8 & 9 \\
\hline Growth at $\mathrm{pH}$ & $5-12$ & $4-12$ & $5-11$ & $5-11$ & $5-12$ & $5-12$ & $5-12$ & $5-12$ & $5-12$ & $5-12$ & $5-12$ & $5-12$ & $5-12$ & $5-12$ \\
\hline \multicolumn{15}{|l|}{ Utilization of: } \\
\hline L-Arabinose & + & + & + & + & + & + & + & + & $+(-1)$ & w & - & - & $+(-1)$ & w \\
\hline D-Fructose & $+(w 1)$ & - & - & + & + & + & - & + & $+(w 1)$ & + & - & w & + & + \\
\hline D-Glucose & + & + & + & + & + & + & + & + & + & + & + & + & + & + \\
\hline myo-Inositol & + & + & $+(w 1)$ & + & + & w & - & + & + & + & + & + & $-(+1)$ & + \\
\hline D-Mannitol & + & + & + & + & + & + & - & + & + & + & + & + & + & + \\
\hline Melezitose & - & - & - & - & - & - & - & - & - & - & - & - & - & - \\
\hline D-Melibiose & - & + & $-(w 1)$ & - & + & + & + & - & $-(+1)$ & + & + & + & $+(-1)$ & w \\
\hline Raffinose & - & + & w & w & + & w & + & - & $-(w 1)$ & w & + & w & $+(-2)$ & $\mathrm{w}$ \\
\hline L-Rhamnose & + & + & + & + & - & + & + & + & + & + & - & + & $+(-1)$ & w \\
\hline D-Sorbitol & - & - & - & - & - & - & - & - & - & - & + & - & - & - \\
\hline Sucrose & - & w & - & w & + & - & - & - & - & w & - & - & - & - \\
\hline D-Xylose & $+(w 1)$ & + & - & + & + & $\mathrm{w}$ & + & + & $+(-1)$ & + & - & - & + & w \\
\hline
\end{tabular}

+ , positive; -, negative; w, weakly positive. Number in parentheses indicates the number of isolate shows positive, weakly positive or negative reaction. 
Table 4: Antimicrobial activity of Streptomyces isolates in Group 1 to Group 14.

\begin{tabular}{|c|c|c|c|c|}
\hline \multirow{2}{*}{ Group } & \multirow{2}{*}{ Isolates } & \multirow{2}{*}{ Nearest relatives (\% identity) } & \multicolumn{2}{|c|}{ Inhibition zone (mm) } \\
\hline & & & 51 production medium & 53 production medium \\
\hline \multirow{5}{*}{1} & KC-054 & S. tendae ATCC $19812^{\mathrm{T}}(100)$ & 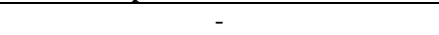 & - \\
\hline & KC-066 & S. tendae ATCC $19812^{\mathrm{T}}(100)$ & $\mathrm{B}(14), \mathrm{K}(14)$ & $\mathrm{B}(9)$ \\
\hline & KC-074 & S. tendae ATCC $19812^{\mathrm{T}}(100)$ & - & - \\
\hline & KC-075 & S. tendae ATCC $19812^{\mathrm{T}}(100)$ & $\mathrm{B}(13), \mathrm{K}(13)$ & $\mathrm{B}(10), \mathrm{K}(10)$ \\
\hline & KC-076 & S. tendae ATCC $19812^{\mathrm{T}}(100)$ & - & - \\
\hline \multirow[b]{2}{*}{2} & KC-060 & S. malachitospinus NBRC $101004^{\mathrm{T}}(99.8)$ & - & $\mathrm{B}(10), \mathrm{X}(11)$ \\
\hline & KC-088 & S. malachitospinus NBRC $101004^{\mathrm{T}}(99.9)$ & - & - \\
\hline \multirow[b]{2}{*}{3} & KC-073 & S. marokkonensis Ap1 $1^{\mathrm{T}}$ (99.4) & $\mathrm{B}(13), \mathrm{K}(9)$ & $\mathrm{B}(11), \mathrm{K}(11)$ \\
\hline & $\mathrm{KC}-155$ & S. marokkonensis Ap $1^{\mathrm{T}}$ (99.5) & $\mathrm{B}(15), \mathrm{K}(14)$ & $\mathrm{K}(9)$ \\
\hline \multirow[b]{2}{*}{4} & KC-055 & S. parvulus NBRC $13193^{\mathrm{T}}(100)$ & $\mathrm{B}(17), \mathrm{K}(22), \mathrm{X}(13)$ & $\mathrm{B}(18), \mathrm{K}(27), \mathrm{X}(15)$ \\
\hline & KC-090 & S. parvulus NBRC $13193^{\mathrm{T}}(100)$ & $\mathrm{B}(25), \mathrm{K}(27), \mathrm{X}(22)$ & $\mathrm{B}(20), \mathrm{K}(18), \mathrm{X}(11)$ \\
\hline 5 & KC-072 & S. fragilis NRRL $2424^{\mathrm{T}}(99.3)$ & - & - \\
\hline 6 & KC-070 & S. diastaticus subsp. ardesiacus NRRL B-1773 ${ }^{\mathrm{T}}$ (99.7) & - & - \\
\hline 7 & KC-085 & S. drozdowiczii NBRC $101007^{\mathrm{T}}(99.4)$ & - & - \\
\hline 8 & KC-156 & S. olivochromogenes NBRC $3178^{\mathrm{T}}(98.4)$ & $\mathrm{B}(11), \mathrm{K}(11)$ & $\mathrm{B}(11), \mathrm{K}(13), \mathrm{E}(12)$ \\
\hline \multirow[b]{2}{*}{9} & KC-141 & S. aureus $\mathrm{NBRC} 100912^{\mathrm{T}}(99.5)$ & $\mathrm{B}(13), \mathrm{K}(13)$ & - \\
\hline & KC-142 & S. aureus $\mathrm{NBRC} 100912^{\mathrm{T}}(99.6)$ & - & - \\
\hline 10 & KC-087 & S. iranensis HM $35^{\mathrm{T}}(99.8)$ & - & $\mathrm{B}(12), \mathrm{K}(15), \mathrm{Ca}(12), \mathrm{Mu}(14)$ \\
\hline 11 & KC-058 & S. rapamycinicus NRRL B-5491 ${ }^{\mathrm{T}}$ (99.3) & $\mathrm{B}(24), \mathrm{K}(27), \mathrm{X}(17), \mathrm{Ca}(17), \mathrm{Mu}(21)$ & $\begin{array}{c}\mathrm{B}(20), \mathrm{K}(22), \mathrm{X}(14), \mathrm{Ca}(16), \\
\mathrm{Mu}(22)\end{array}$ \\
\hline 12 & KC-079 & S. yatensis NBRC $101000^{\mathrm{T}}(99.4)$ & $\mathrm{B}(17), \mathrm{K}(11), \mathrm{X}(15), \mathrm{Ca}(16), \mathrm{Mu}(19)$ & $\mathrm{B}(12), \mathrm{K}(15), \mathrm{Ca}(12), \mathrm{Mu}(14)$ \\
\hline \multirow{4}{*}{13} & KC-080 & S. samsunensis $\mathrm{M} 1463^{\mathrm{T}}(99.9)$ & $\mathrm{B}(14), \mathrm{K}(12), \mathrm{Ca}(9), \mathrm{Mu}(10)$ & $\mathrm{B}(11), \mathrm{K}(10), \mathrm{Ca}(15), \mathrm{Mu}(11)$ \\
\hline & KC-093 & S. samsunensis $\mathrm{M} 1463^{\mathrm{T}}(100)$ & $\mathrm{B}(15), \mathrm{K}(12), \mathrm{Ca}(13), \mathrm{Mu}(19)$ & $\mathrm{B}(10), \mathrm{K}(10), \mathrm{Ca}(13), \mathrm{Mu}(18)$ \\
\hline & KC-094 & S. samsunensis $\mathrm{M} 1463^{\mathrm{T}}(100)$ & $\mathrm{B}(18), \mathrm{K}(15), \mathrm{Ca}(15), \mathrm{Mu}(18)$ & $\mathrm{B}(10), \mathrm{K}(11), \mathrm{Ca}(15), \mathrm{Mu}(15)$ \\
\hline & $\mathrm{KC}-151$ & S. samsunensis $\mathrm{M} 1463^{\mathrm{T}}(100)$ & $\mathrm{B}(10), \mathrm{Ca}(11), \mathrm{Mu}(11)$ & - \\
\hline \multirow{2}{*}{14} & KC-062 & S. spiralis NBRC $14215^{\mathrm{T}}(99.4)$ & - & $\mathrm{B}(10), \mathrm{X}(11)$ \\
\hline & KC-063 & S. spiralis NBRC $14215^{\mathrm{T}}(99.0)$ & - & $\mathrm{B}(10), \mathrm{K}(14)$ \\
\hline
\end{tabular}

\section{CONCLUSION}

In conclusion, twenty-six strains of Streptomyces were isolated from thirteen soil samples collected around Angthong Islands National Park, Surat Thani province, the southern part of Thailand. They were identified as $S$. tendae, S. malachitospinus, S. marokkonensis, S. parvulus, S. fragilis, S. diastaticus, $S$. drozdowiczii, S. olivochromogenes, S. aureus, S. iranensis, S. rapamycinicus, S. yatensis, S. samsunensis, and $S$. spiralis based on their morphological, cultural, physiological and biochemical characteristics including the $16 \mathrm{~S}$ rRNA gene sequence analysis. They exhibited antimicrobial activity against $B$. subtilis ATCC 6633, K. rhizophila ATCC 9341, M. racemosus IFO 4581, C. albicans KF1, E. coli NIHJ KB213 and X. campestris pv. oryzae KB88. Our strains in $S$. malachitospinus, $S$. marokkonensis, S. drozdowiczii, S. aureus, S. iranensis, $S$. yatensis, S. samsunensis, and S. spiralis are required for further study on their bioactive compounds.

\section{ACKNOWLEDGEMENTS}

This study was supported by Chulalongkorn University Dutsadi Phiphat Scholarship (2008) for a Ph.D. Program to P. S. We thank Prof. Dr. Yoko Takahashi, Dr. Atsuko Matsumoto, Dr.Yuki Inahashi and the staffs of Kitasato Institute for Life Sciences, Kitasato University, Tokyo, Japan for supporting the screening and sequencing of the isolates.

\section{REFERENCES}

Arumugam M, Mitra A, Pramanik A, Saha M, Gachhui R, Mukherjee J. Streptomyces sundarbansensis sp. nov., an actinomycete that produces 2-allyloxyphenol, Int J Syst Evol Microbiol, 2011; 61: 2664-69.

Baranasic D, Gacesa R, Starcevic A, Zucko J, Blažič M, Horvat M, Gjuračić K, Fujs Š, Hranueli D, Kosec G, Cullum J, Petković H. Draft genome sequence of Streptomyces rapamycinicus strain NRRL 5491, the producer of the immunosuppressant rapamycin. Genome Announc, 2013; 1(4): e00581-13

Berdy J. Bioactive microbial metabolites: Review article. J Antibiot, 2005; 58:1-26.

Bormann C, Baier D, Hörr I, Raps C, Berger J, Jung G, Schwarz H. Characterization of a Novel, Antifungal, chitin-binding protein from Streptomyces tendae Tü901 that interferes with growth polarity. J Bacteriol, 1999; 181(24) 7421-7429.

Bouizgarne B, Lanoot B, Loqman S, Sproer C, Klenk HP, Swings J, Ouhdouch Y. Streptomyces marokkonensis sp. nov., isolated from rhizosphere soil of Argania spinosa L. Int J Syst Evol Microbiol, 2009; 59:2857-2863.

Faulds CB, Williamson G. The purification and characterization of 4-hydroxy-3-methoxycinnamic (ferulic) acid esterase from Streptomyces olivochromogenes. J Gen Microbiol, 1991; 137(10): 2339 2345.

Glasby JS. 1993. Encyclopedia of Antibiotics. $3^{\text {rd }}$ ed. John Wiley and Sons, Chichester, p. 1-507.

Gouy M, Gascuel S, Gascuel O. SeaView version 4.2: a multiplateform graphical user interface for sequence alignment and phylogenetic tree building. Mol Biol Evol, 2010; 27:221-224.

Goodfellow M, Williams ST, Mordarski M. 1988 Actinomycetes in Biotechnology, London: Academic Press Inc, p. 1-88.

Hamedi J, Mohammadipanah F, Klenk HP, Pötter G, Schumann P, Spröer C, Vonjan M, Kroppenstedt RM. Streptomyces iranensis sp. nov., isolated from soil. Int J Syst Evol Microbiol, 2010; 60:1504-1509. 
Kämpfer P . 2012. Genus Streptomyces: The Actinobacteria In: Goodfellow M, Kämpfer P, Busse H-J, Trujillo, ME, Suzuki K, Ludwig W, Whitman, WB, eds. Bergey's Manual of Systematic Bacteriology, 2nd Edition, Part B, New York: Springer, p.1455-1767.

Kalakoutskii LV, Zenova GM, Soina VS, Likhacheva AA. Associations of actinomycetes with algae. Actinomycetes, 1990; 1: 27-42.

Kim OS, Cho YJ, Lee K, Yoon SH, Kim M, Na H, Park SC, Jeon YS, Lee JH, Yi H, Won S, Chun J. Introducing EzTaxon-e: a prokaryotic 16S rRNA gene sequence database with phylotypes that represent uncultured species. Int J Syst Evol Microbiol, 2012; 62: 716721.

Kimura MA simple method for estimating evolutionary rates of base substitutions through comparative studies for nucleotide sequences. J Mol Evol, 1980; 16:111-120.

Kumar Y, Goodfellow M. Five new members of the Streptomyces violaceusniger $16 \mathrm{~S}$ rRNA gene clade: Streptomyces castelarensis sp. nov., comb. nov., Streptomyces himastatinicus sp. nov., Streptomyces mordarskii sp. nov., Streptomyces rapamycinicus sp. nov. and Streptomyces ruanii sp. nov. Int J Syst Evol Microbiol, 2008; 58:1369-1378.

Lorain V. (1991). Antibiotics in Laboratory Medicine, Baltimore: The Williams \& Wilkins Co., p. 1-51.

Manfio GP, Atalan E, Zakrzewska CJ, Mordarski M, Rodriguez C, Collins MD, Goodfellow M. Classification of novel soil streptomycetes as Streptomyces aureus sp. nov., Streptomyces laceyi sp. nov. and Streptomyces sanglieri sp. nov. Ant Van Leeuwenhoek, 2003; 85:24555 .

Matsumoto A, Takahashi Y, Iwai Y, Omura S. Isolation of Gram-positive bacteria with high $\mathrm{G}+\mathrm{C}$ from inside soil aggregates. Actinomycetologica, 2006; 20:30-40.

Mundie DA. (1995). The NBS/ISCC Color System / David A. Mundie Pittsburgh, PA: Polymath Systems 535.6 dc-20 Available at: http://www.dodomagnifico.com/Colors/NBS.html. Accessed June 5, 2011.

Pittillo RF, Hunt DE. Azaserine and 6-diazo-5-oxo-Lnorleucine (DON). J Antibiot, 1967; 1: 481-493.

Reddy TV, Mahmood S, Paris L, Reddy YH, Wellington EM, Idris MM. Streptomyces hyderabadensis sp. nov., an actinomycete isolated from soil. Int J Syst Evol Microbiol, 2011; 61:76-80.

Roos U, Mattern S, Schrempf H, Bormann C. Histidine aminotransferase activity in Streptomyces tendae and its correlation with nikkomycin production. FEMS Microbiol Lett, 1992; 76: 185-190.

Saintpierre D, Amir H, Pineau R, Sembiring L, Goodfellow M. Streptomyces yatensis sp. nov., a novel bioactive streptomycete isolated from a new-caledonian ultramafic soil. Antonie Van Leeuwenhoek, 2003; 83(1):21-26.

Saito N, Nei M. The neighbor-joining method: a new method for reconstructing phylogenetic trees. Mol Biol Evol, 1987; 4:406-425.

Seco EM, Cuesta T, Fotso S, Laatsch H, Malpartida F. Two polyene amides produced by genetically modified Streptomyces diastaticus var. 108, Chem Biol, 2005; 12 (5): 535-543.
Semedo LT, Gomes RC, Linhares AA, Duarte GF, Nascimento RP, Rosado AS, Margis PM, Margis R, Silva KR, Alviano CS, Manfio GP, Soares RM, Linhares LF, Coelho RR. Streptomyces drozdowiczii sp. nov., a novel cellulolytic streptomycete from soil in Brazil. Int J Syst Evol Microbiol, 2004; 54:1323-1328.

Santhanam R, Rong X, Huang Y, Andrews BA, Asenjo JA, Goodfellow M. Streptomyces bullii sp. nov., isolated from a hyper-arid Atacama Desert soil. Antonie Van Leeuwenhoek, 2013; 103(2):367-373.

Sazak A, Sahin N, Guven K, Isik K, Goodfellow M. Streptomyces samsunensis sp. nov., a member of the Streptomyces violaceusniger clade isolated from the rhizosphere of Robinia pseudoacacia. Int J Syst Evol Microbiol, 2011; 61:1309-1314.

Seong CN, Choi JH, Baik KS. An improved selective isolation of rare actinomycetes from forest soil. J Microbiol, 2001; 39:17-23.

Shetty PR, Buddana SK, Tatipamula VB, Naga YVV, Ahmad J. Production of polypeptide antibiotic from Streptomyces parvulus and its antibacterial activity. Braz J Microbiol, 2014; 45(1): 303-312.

Sripreechasak P, Matsumoto A, Suwanborirux K, Inahashi Y, Shiomi K, Tanasupawat S, Takahashi Y. Streptomyces siamensis sp. nov., and Streptomyces similanensis sp. nov., isolated from Thai soils. J Antibiot, 2013a, 66(11):633-640.

Sripreechasak P, Suwanborirux K, Inahashi Y, Matsumoto A, Tanasupawat S, Takahashi Y. Identification and antimicrobial activity of actinobacteria from soils in southern Thailand. Trop Biomed, 2013b; 30(1): 46-55.

Shirling EB, Gottlieb D. Methods for characterization of Streptomyces species. Int J Syst Bacteriol, 1966; 16:313-340.

Syed DG, Agasar D, Kim CJ, Li WJ, Lee JC, Park DJ, Xu LH, Tian XP, Jiang CL. Streptomyces tritolerans sp. nov., a novel actinomycete isolated from soil in Karnataka, India. Antonie Van Leeuwenhoek, 2007; 92(4):391-397.

Takahashi Y, Matsumoto A, Seino A, Ueno J, Iwai Y, Omura S. Streptomyces avermectinius sp. nov., an avermectin-producing strain. Int J Syst Evol Microbiol, 2002; 52:2163-2168.

Tajima K, Takahashi Y, Seino A, Iwai Y, Omura S. Description of two novel species of the genus Kitasatospora, Kitasatospora cineracea sp. nov. and Kitasatospora niigatensis sp. nov. Int J Syst Evol Microbiol, 2001; 51: 1765-1771.

Yang PW, Li MG, Zhao JY, Zhu MZ, Shang H, Li JR, Cui XL, Huang R, Wen ML. Oligomycins A and C, major secondary metabolites isolated from the newly isolated strain Streptomyces diastaticus. Folia Microbiol, 2010; 55(1):10-16.

\section{How to cite this article:}

Paranee Sripreechasak, Khanit Suwanborirux and Somboon Tanasupawat. Characterization and antimicrobial activity of Streptomyces strains from soils in southern Thailand. J App Pharm Sci, 2014; 4 (10): 024-031. 